\title{
The immune cell landscape of metastatic uveal melanoma correlates with overall survival
}

\author{
Anna Tosi ${ }^{1}$, Rocco Cappellesso ${ }^{2}$, Angelo Paolo Dei Tos ${ }^{2,3}$, Valentina Rossi ${ }^{4}$, Camillo Aliberti ${ }^{5}$, Jacopo Pigozzo ${ }^{6}$, \\ Alessio Fabozzi ${ }^{7}$, Marta Sbaraglia², Stella Blandamura ${ }^{3}$, Paola Del Bianco ${ }^{8}$, Vanna Chiarion-Sileni ${ }^{6+}$ and \\ Antonio Rosato ${ }^{1,4^{*}+}$ (D)
}

\begin{abstract}
Background: Uveal melanoma (UM) represents the most common primary intra-ocular malignancy in adults. Up to 50\% of the patients develop distant metastases within 10 years from diagnosis, with the liver as the most common site. Upon metastatization, life expectancy strongly reduces and immune checkpoint inhibitors that prove effective in cutaneous melanoma do not modify clinical outcome. To date, few studies have focused on deciphering the immunomodulatory features of metastatic UM microenvironment, and there are no prognostic models for clinical use. This highlights the urgent need to understand the delicate interplay between tumor and immune cells acting at the site of metastasis.

Methods: We collected a patient cohort comprising 21 metastatic UM patients. Hepatic and extra-hepatic UM metastasis samples were studied by multiplex immunofluorescence to assess the tumor immune cell composition. Quantitative analyses were performed to correlate immune cell densities with treatment response, metastasis site and patient survival.

Results: Compared to patients with progressive disease, those with controlled disease had a higher intra-tumoral/ peritumoral ratio of CD8 + Granzyme B+ cells, higher density of intra-tumoral CD8+ cytotoxic T lymphocytes (CTL) and an increased percentage of UM cells in close proximity to T lymphocytes, reflecting a role of tumor-killing $T$ cells in the disease. In liver metastases (LM), the intra-tumoral densities of CD163+ tumor-associated macrophages (TAM) and of total CD8+ T cells were higher than in extra-hepatic UM metastases, but the percentage of Granzyme B+ CTL was lower. Moreover, LM displayed more UM cells adjacent to both CTL and TAM, and also more T cells in proximity to TAM, all signs of an impaired immune response. The percentage of activated $C T L$ within the tumor represented a prognostic indicator, as patients with a higher intra-tumoral percentage of CD8 + Granzyme B+ cells had the better outcome. A temptative Immunoscore was generated and proved capable to stratify patients with improved survival. Finally, CD4 + FoxP3+ T cells appeared a crucial population for response to immunotherapy.
\end{abstract}

Conclusion: The results of this study underly the clinical relevance and functional importance of composition and localization of antitumor effector cells for the progression of UM metastasis.

Keywords: Metastatic uveal melanoma, Tumor microenvironment, Immune cell, Immune contexture, Immune biomarkers

\footnotetext{
* Correspondence: antonio.rosato@unipd.it

${ }^{\dagger}$ Antonio Rosato and Vanna Chiarion-Sileni contributed equally as last author

'Department of Surgery, Oncology and Gastroenterology, University of

Padova, Padova, Italy

${ }^{4}$ Immunology and Molecular Oncology Diagnostics, Veneto Institute of

Oncology IOV-IRCCS, Via Gattamelata 64, 35128 Padova, Italy

Full list of author information is available at the end of the article
}

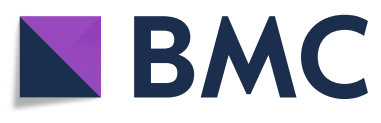

(c) The Author(s). 2021 Open Access This article is licensed under a Creative Commons Attribution 4.0 International License, which permits use, sharing, adaptation, distribution and reproduction in any medium or format, as long as you give appropriate credit to the original author(s) and the source, provide a link to the Creative Commons licence, and indicate if changes were made. The images or other third party material in this article are included in the article's Creative Commons licence, unless indicated otherwise in a credit line to the material. If material is not included in the article's Creative Commons licence and your intended use is not permitted by statutory regulation or exceeds the permitted use, you will need to obtain permission directly from the copyright holder. To view a copy of this licence, visit http://creativecommons.org/licenses/by/4.0/. The Creative Commons Public Domain Dedication waiver (http://creativecommons.org/publicdomain/zero/1.0/) applies to the data made available in this article, unless otherwise stated in a credit line to the data. 


\section{Background}

Uveal melanoma (UM) originates from the uveal tract of the eye [1], and differs from the cutaneous melanoma in risk factors, biological behavior, epidemiology, prognostic features, and molecular profiles. Indeed, UM has an extremely low mutational burden contrary to cutaneous melanoma [2], which harbors a high rate of $\mathrm{C}>\mathrm{T}$ transitions and double CC > TT mutations induced by UV exposure, and hot-spot mutations in $B R A F$ or $R A S$ or loss of function mutations in NF1 [3]. On the other hand, the vast majority (85-95\%) of metastatic UM harbors monosomy 3, GNA11 or GNAQ mutations [4]. Despite the improvement and effectiveness of local tumor control ( $80 \%$ at 5 years), the high tendency to metastasize has not changed [5] and still up to $50 \%$ of the patients develop distant metastases within 10 years after diagnosis. The liver is the most common site (80-90\%), followed by lung $(29 \%)$ and bone $(17 \%)[6,7]$. Once metastases are present, the disease course is generally aggressive and the prognosis remains poor, with a median overall survival (OS) of 13.4 months [8], and a 2-year OS rate of only $8 \%$ [9].

Liver metastasis is a relevant aspect of clinical course, and liver failure is almost the exclusive cause of death even when other visceral sites are involved. The median survival of patients who develop UM liver metastasis ranges between 6 to 12 months, as compared to 19-28 months for patients who first metastasize in other sites $[6,10,11]$. In the metastatic stage, UM systemic therapy largely derives from approaches effective against cutaneous melanoma. Additionally, a variety of local liverdirected treatment options have been investigated, including surgical resection, hepatic artery embolization, hepatic arterial chemotherapy infusion, and radiofrequency, but none of them has resulted in an improved survival in metastatic disease [12]. Further, CTLA-4 (ipilimumab) and PD-1 (nivolumab, pembrolizumab) inhibitors as monotherapy in sequence or combined, and targeted therapies with anti-angiogenic and kinase inhibitors [13] have been also tested, but with disappointing results or only marginal success to date [14-16]. Recently, the adoptive transfer of in vitro expanded autologous tumor-infiltrating lymphocytes (TILs) has been reported to mediate objective tumor regression in some patients with metastatic UM, thus fostering further investigation on the role of immune cells in this challenging disease [17].

Assays using a variety of molecular techniques have the ability to analyze the primary tumor to predict prognosis and the risk of metastasis [18, 19]. Differently from what observed in other cancer types [20], evaluation of the prognostic impact of immune system in primary UM has revealed that high densities of tumor-associated macrophages (TAMs) and TILs are associated with a poor prognosis, and a high risk of metastasis [21-23]. However, few studies have focused on deciphering the immunomodulatory features of metastatic UM microenvironment [24, 25], largely due to the difficulty in acquiring specimens that often derive from percutaneous biopsies. Moreover, to date there are no prognostic models for clinical use in newly diagnosed metastatic disease [26, 27]. Circulating UM cells that enter the liver encounter a unique immune system, as liver also acts as an immune-modulating organ devoted to quickly defeat gastrointestinal-derived pathogens, and at the same time to maintain tolerance against harmless food antigens [28]. Therefore, interaction between liver immune system and cancer cells provide a complex tumor microenvironment that could help UM cells to evade an antitumor immune response. Thus, efforts must be put in place to understand the delicate interplay that occurs between tumor and immune cells acting at the site of metastasis, to allow the identification of prognostic/predictive factors which could facilitate the tailored management of patients and improve survival outcomes.

We designed a study aimed at analysing the density and the spatial distribution of immune cell subpopulations in a cohort of patients with hepatic and extrahepatic metastasis from UM. The final goal was to identify immune biomarkers able to capture the immune contexture of tumor microenvironment that could stratify patients with better prognosis, in order to guide patient care and to facilitate future rational trial design to target appropriate metastatic UM patient subgroups.

\section{Methods}

\section{Patient characteristics}

The study cohort comprised 21 patients diagnosed with metastatic UM and treated at Veneto Institute of Oncology IOV-IRCCS in Padova, from March 2006 to July 2019. Last follow-up date was September 2020. The biopsies of the 17 liver and 4 extra-hepatic UM metastases included in this study were obtained prior the beginning of treatments. After the diagnosis of UM metastasis, the sequence of treatments varied according to the period of the treatment, the availability of immune checkpoint inhibitors in Italy, the sites of metastases and the clinical conditions of the patients. All patients with a prevalent disease burden in the liver received transcatheter arterial chemoembolization with microbeads charged with irinotecan $100 \mathrm{mg}$ (DEBIRI-TACE). The procedure was repeated 6 weeks later in all patients, and thereafter every 3-6 months according to the disease evaluation. Systemic therapies were administered concomitantly to the liver-directed treatments avoiding the 4 days before and the 10 days after DEBIRI-TACE, to limit the risk of an increased liver toxicity. All participants signed a written informed consent form to allow the use of their 
diagnostic tumor biopsy for the assessment of tumor immune-microenvironment, and its correlation with the clinical tumor response and outcome. The study was conducted at the Veneto Institute of Oncology IOVIRCCS in accordance with Good Clinal Practices, local regulatory requirements, and Declaration oh Helsinki.

\section{Tissue samples}

All cases were reviewed and the diagnoses confirmed in all instances by an expert pathologist. Consecutive $4 \mu \mathrm{m}$ thick sections were cut from formalin-fixed and paraffinembedded (FFPE) tissue block of each case for subsequent analyses.

\section{Multiplex fluorescence immunohistochemistry (mIHC)} mIHC staining was performed using the Opal seven-color manual kit (Akoya Biosciences) following the manufacturer's instructions, as previously reported [29]. Two staining panels were used to characterize the subsets of tumorinfiltrating immune cells, and the list of antibodies used is summarized in Table 1. All stains were performed under optimized conditions. Before proceeding with multiplex experiments, the optimal staining conditions for each marker of the panel were determined using monoplex stained slides from a positive control tissue (human tonsil). Thus, the antigen-fluorophore pairing (according to the expression characteristics of each specific antigen), the order of primary antibodies addition to the panel (based on the epitope biology and sensitivity), and the concentration and incubation time of primary antibodies and fluorophores were optimized. These parameters were then revaluated in a multiplex stained metastatic UM sample slide, and the optimized multiplex staining protocol was then applied to all sample slides. Moreover, an unstained tissue section was used to subtract the tissue autofluorescence from multiplex-stained slides.

\section{Multispectral imaging and analysis}

Multiplex stained slides were imaged using the Mantra Quantitative Pathology Workstation (Akoya Biosciences) at 20X magnification (Fig. 1a). For each sample, only areas comprising tumor cells were considered, and at least 20 fields at $20 \mathrm{X}$ magnification were acquired for each slide in order to encompass all UM metastasis regions avoiding overlaps between tissue fields. The inForm Image Analysis software (version 2.4.9, Akoya Biosciences) was used to unmix multispectral images using a spectral library built from acquisition of single fluorophore-stained control tissues and containing fluorophores-emitting spectral peaks. A selection of representative multispectral images was used to train the inForm software to create an algorithm for each panel. Tumor tissue was segmented based on recognition of cells stained positive for the anti-melanoma antibody cocktail, to differentiate infiltrating immune cells within the tumor area and in the surrounding stroma (Fig. 1b, left). Then, nuclear counterstaining was used to segmented single cells (Fig. 1b, middle) and cell phenotyping was based on the detection of specific cell-surface or intracellular markers (Fig. 1b, right). The created algorithms were applied in the batch analysis of all acquired seven-color multispectral images of the same panel. Cell densities and percentages were calculated for each patient as the mean of all acquired field of the same tissue slide (at least 20 fields at 20X magnification for each stained slide).

\section{Cell-to-cell distance analysis}

Topographic coordinates for each cell within each tissue section were obtained by InForm software, and distance analyses were performed using Phenoptr and Phenoptr Reports (add-ins for R Studio from Akoya Biosciences). For mean cell distance between tumor cells and the nearest $\mathrm{T}$ lymphocyte, the nearest neighbor analysis was used (Fig. 1c), while count within analysis was employed to calculate the percentage of reference cells (tumor or immune cells) within a specified $\mu \mathrm{m}$ radius from a specific immune cell subtype, among the total number of reference cells (Fig. 1d).

\section{Statistical analysis}

All statistical analyses were performed using the GraphPad Prism software (version 7.0). Disease control rate (DCR) was defined as the proportion of patients with stable disease and complete/partial response to treatment, according to RECIST 1.1 criteria [30]. For continuous variables, median, quartiles and range were described and statistical analyses were performed with the non-parametric two-tailed Mann-Whitney test between groups of interest. OS was the time from metastatic UM diagnosis to death. Patients who did not develop an event during the study period were censored at the date of last follow up. For survival analyses, each marker was categorized as low or high according to its median value. The survival probabilities were estimated by the Kaplan-Meier method, and compared between the marker groups using the log-rank Mantel-Cox test. Results were expressed as hazard ratios (HR) with their 95\% confidence intervals (95\% CI), obtained from a Cox Proportional Hazards model. For the correlation analyses, the non-parametric Spearman's correlation coefficient ( $\mathrm{r}$ ) was calculated. A $p$-value $\leq 0.05$ was considered statistically significant.

\section{Results}

Patients characteristics

The characteristics of the 21 patients are summarized in Table 2. Fifteen patients (71.4\%) developed metastases 
Table 1 List of primary antibodies used in $\mathrm{mlHC}$ staining

\begin{tabular}{llll}
\hline Antigen & Panel & Clone & Vendor \\
\hline Neutrophil Elastase (NE) & 1st & NP57 & Dako \\
CD56 & 1 st & NP57 & Dako \\
CD3 & $1 s t$ & F.7.2.38 & Dako \\
CD20 & $1 s t$ & L26 & Dako \\
CD68 & 1 st & KP1 & Dako \\
CD8 & 2nd & C8/144B & Dako \\
CD4 & 2nd & $4 B 12$ & ThermoFisher \\
Granzyme B & 2nd & $11 F 1$ & Leica Biosystems \\
FoxP3 & 2nd & D2W8E & Cell Signalling \\
CD163 & 2nd & $10 D 6$ & Leica Biosystems \\
Anti-melanoma mix (HMB-45 + Mart-1 + Tyrosinase + SOX-10) & 1st and 2nd & HMD45 + M2-7C10 + M2-9E3 + T311 + EP268 & Abcam and Cell Marque \\
Spectral DAPl & 1st and 2nd & Akoya Biosciences \\
\hline
\end{tabular}

confined to the liver, two patients $(9.6 \%)$ metastasized also in other sites (but only their liver metastases could be evaluated), while in four patients (19\%) only extrahepatic sites were involved. About this latter group, only ureter, lymph node, small intestine and thigh metastases were collected and analyzed. In the study cohort, 13 patients $(61.9 \%)$ were treated with checkpoint inhibitors immunotherapy, while 8 patients (38.1\%) with other targeted/systemic therapies. At a median follow-up of 25.8 months (range 6.8-171) from metastatic UM diagnosis, 11 patients $(52.4 \%)$ were alive with residual disease, while 10 (47.6\%) were dead. Median OS from metastatic UM diagnosis was 95.7 months. Seven patients (33.3\%) had progressive disease, 7 patients (33.3\%) stable disease, 6 patients $(28.6 \%)$ reached a partial response and 1 patient $(4.8 \%)$ obtained a complete response.

The immune microenvironment of UM metastases differs between patients with progressive (PD) and controlled (CD) disease

A mIHC approach was applied to FFPE tissue sections of metastatic UM. Two panels of representative immune markers were designed to describe the composition of immune cells infiltrating the metastasis microenvironment. The first panel included CD20, CD3, CD68, CD56 and NE as markers of B lymphocytes, $\mathrm{T}$ cells, macrophages, NK cells and neutrophils, respectively. The purpose of the second panel was to investigate the functional state of immune cells, as it included CD4 as a marker of $\mathrm{T}$ helper lymphocytes, FoxP3 expressed by $\mathrm{CD} 4+\mathrm{T}$ regulatory cells $\left(\mathrm{T}_{\text {reg }}, \mathrm{CD} 4+\right.$ FoxP3+), $\mathrm{CD} 8$ for cytotoxic $\mathrm{T}$ lymphocytes (CTL), granzyme $\mathrm{B}$ to identify antitumor activated CTL (CD8+/CD4+ Granzyme B+), and CD163 recapitulating M2-polarized TAM. In both panels, a cocktail of anti-melanoma antigen-recognizing antibodies was added to outline tumor cells (Fig. 2a).
Patients were divided into two subgroups according to the disease control rate: patients with progressive disease (PD, $n=7$ ) versus those who had response or stable disease (CD, $n=14)$. In either groups, CD3+ T cells constituted the dominant TIL subset, while CD56+ NK cells and NE+ neutrophils appeared negligible (Fig. 2b, upper panels); CD68+ macrophages were the major cell fraction at intra-tumoral level, being less represented in the peritumoral stroma (Fig. 2b, upper panels).

Notwithstanding, a deeper analysis of macrophage and $\mathrm{T}$ cell subpopulations revealed that patients with $\mathrm{CD}$ had a lower fraction of CD163+ M2-polarized macrophages and a higher percentage of total CD8+ T lymphocytes than PD patients, both in the intra-tumoral area and in the surrounding stroma (Fig. 2b, middle panels). As the CD8/CD4 ratio is often used as a simple measure to determine the overall balance of $\mathrm{T}$ cell function in cancer [31, 32], such lymphocyte subsets were specifically quantified. The total CD4+ T cell component was substantially similar in PD and CD patients irrespectively of the tumor/stroma location; therefore, the CD8/ $\mathrm{CD} 4 \mathrm{~T}$ cell ratio differed between the two groups, being higher in CD patients mainly in the stromal area (Fig. 2b, middle panels and Fig. 2c). Apart quantitative aspects, sample tissue staining with the second mIHC antibody panel provided further insights about the functional state of $\mathrm{T}$ lymphocytes present in the metastatic microenvironment. CD patients had a lower percentage of CD4+ FoxP3 $+\mathrm{T}_{\text {reg }}$ cells than PD counterparts both in the intra-tumoral and stromal regions, while no difference was observed in the CD4 + Granzyme B+ subset (Fig. 2b, bottom panels). As tumor infiltration by activated CD8+ $\mathrm{T}$ lymphocytes is considered a feature associated with tumor-specific immune response, the microenvironment localization of such population was investigated. The CD8 + Granzyme B+ CTL fraction was percentually similar in the intra-tumoral area of either patient groups, 


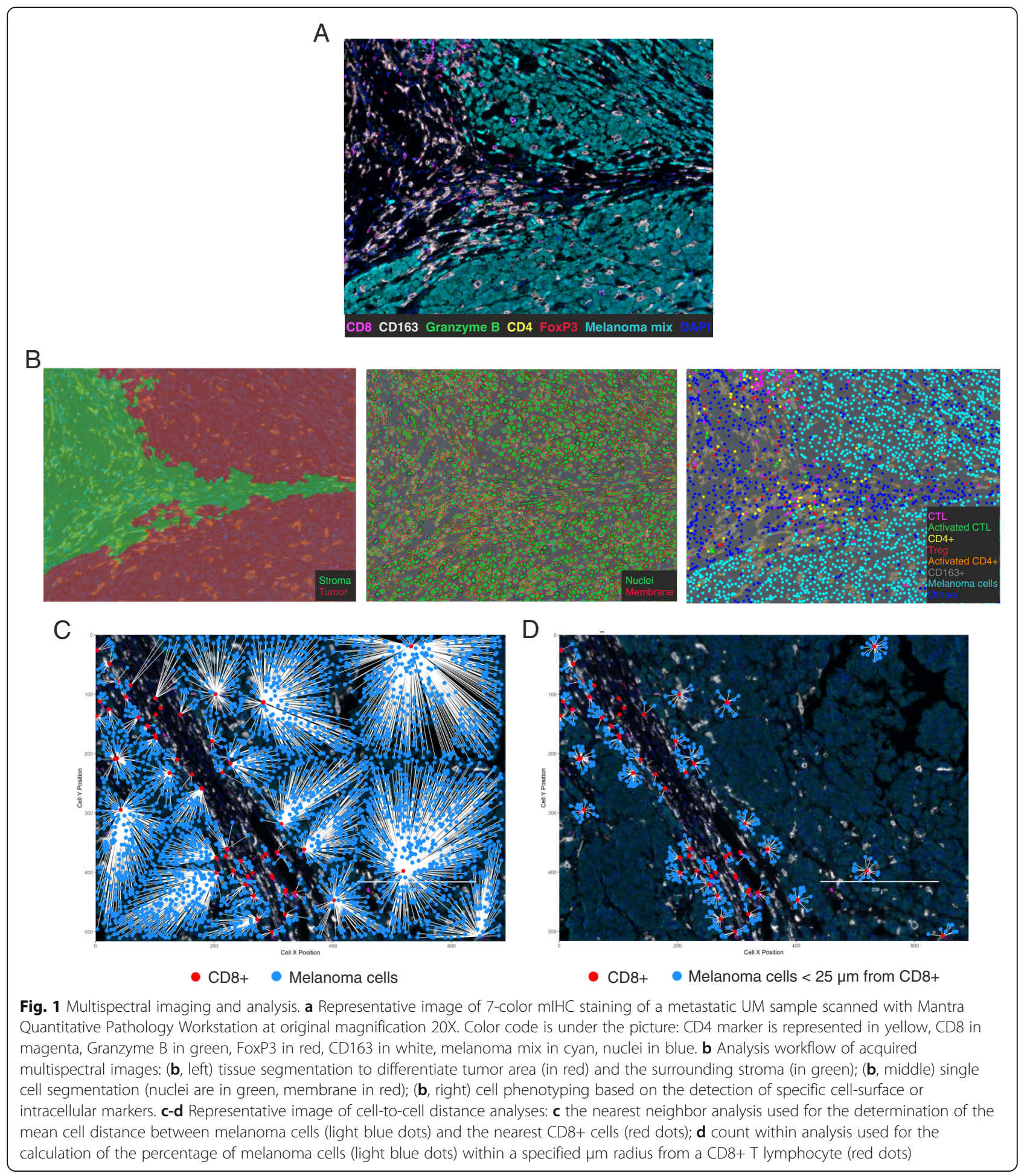

but was globally higher in patients with PD whether considering the surrounding stroma (Fig. 2b, bottom panels). On the other hand, a higher CD8 + Granzyme $\mathrm{B}+\mathrm{T}$ cell intra-tumoral/peritumoral (I/P) ratio was observed in $C D$ patients (Fig. 2d), suggesting their preferential infiltration of tumor masses where they might be regarded as exerting a direct cytotoxic activity against metastatic UM cells.

Additionally, we calculated the densities of immune cell subsets infiltrating the UM metastases in different areas of tumor microenvironment. No differences were observed in B lymphocytes and myeloid cells (data not 
Table 2 Patient characteristics

\begin{tabular}{|c|c|c|}
\hline & $\mathbf{N}$ & $\%$ \\
\hline \multicolumn{3}{|l|}{ Gender } \\
\hline Female & 9 & 42.9 \\
\hline Male & 12 & 57.1 \\
\hline \multicolumn{3}{|l|}{ Age at diagnosis of primary UM, years } \\
\hline Median (IQR) & $61(49-66)$ & \\
\hline \multicolumn{3}{|l|}{ Age at diagnosis of UM metastasis, years } \\
\hline Median (IQR) & $65(57-70)$ & \\
\hline \multicolumn{3}{|l|}{ Metastasis site } \\
\hline Liver & 17 & 81.0 \\
\hline Extra-hepatic & 4 & 19.0 \\
\hline \multicolumn{3}{|l|}{ Liver involvement ( $n=17), \%$} \\
\hline$<20$ & 8 & 47.1 \\
\hline $20-50$ & 8 & 47.1 \\
\hline$>50$ & 1 & 5.8 \\
\hline \multicolumn{3}{|l|}{ LDH } \\
\hline Normal & 15 & 71.4 \\
\hline$>1 U L N$ & 6 & 28.6 \\
\hline \multicolumn{3}{|l|}{ Treatment } \\
\hline Checkpoint inhibitors immunotherapy & 13 & 61.9 \\
\hline Targeted/Systemic therapies & 8 & 38.1 \\
\hline \multicolumn{3}{|l|}{ Response } \\
\hline PD & 7 & 33.3 \\
\hline SD & 7 & 33.3 \\
\hline$P R$ & 6 & 28.6 \\
\hline$C R$ & 1 & 4.8 \\
\hline \multicolumn{3}{|l|}{ Status } \\
\hline Alive with disease & 11 & 52.4 \\
\hline Dead & 10 & 47.6 \\
\hline
\end{tabular}

Abbreviations: IQR interquartile range, $N$ normal, ULN upper limit of normal, $P D$ progressive disease, $S D$ stable disease, $P R$ partial response, $C D$ complete response. Response evaluation for each patient was in accordance with RECIST $1.1[30]$

shown). However and with regard to the T cell compartment, a higher density of intra-tumoral CD8 $+\mathrm{T}$ cells was observed in patients with CD (Fig. 2e).

To assess whether the increased immune infiltrate might associate to inhibitory or activating characteristics, correlation analyses were performed between functionally different immune cell populations. A direct correlation between intra-tumoral CD4+ T lymphocytes and $\mathrm{CD} 4+$ FoxP3 $+\mathrm{T}_{\text {reg }}$ cell densities was observed in PD patients $(r=0.9316,95 \%$ CI 0.598 to 0.99 ; Supplementary Figure $1 \mathrm{~A})$, while a positive correlation between intratumoral $\mathrm{CD} 4+\mathrm{T}$ cells and the activated $\mathrm{CD} 4+$ Granzyme $\mathrm{B}+$ cell counterpart existed in $\mathrm{CD}$ individuals $(r=$ 0.6492, 95\%CI 0.164 to 0.886; Supplementary Figure 1B), but not in PD patients $(r=-0.4714,95 \% \mathrm{CI}-0.903$ to
0.436; data not shown). Conversely, an inverse correlation between intra-tumoral CD4+ lymphocytes and the percentage of $\mathrm{T}_{\text {reg }}$ cells was detected in CD patients $(r=$ $-0.5867,95 \%$ CI -0.856 to -0.064 ; Supplementary Figure $1 \mathrm{C})$. Moreover, in this latter group, the densities of macrophages (CD68+ and CD163+) and T lymphocytes subpopulations were directly proportional both at intratumoral $(r=0.6879,95 \% \mathrm{CI} 0.231$ to 0.896 and $r=$ $0.7934,95 \%$ CI 0.4399 to 0.934 , respectively; Supplementary Figure 1D) and stromal levels $(r=0.7566,95 \% \mathrm{CI}$ 0.305 to 0.0 .93 and $r=0.936495 \% \mathrm{CI} 0.759$ to 0.984 , respectively; Supplementary Figure 1E). No association between $\mathrm{T}$ cell and macrophage densities was present in PD patients (data not shown). These observations suggest an active immune response that in $C D$ patients is mediated by both $\mathrm{T}$ and myeloid cells.

To explore the spatial distributions and interactions of cancer cells with respect to immune cells, the Cartesian coordinates were calculated for each cell subset within each tissue section, and cell-to-cell distance analyses were performed (Fig. 1c, d). As the close proximity between $\mathrm{T}$ lymphocytes and tumor cells is held as an indicator of an ongoing specific anti-tumor immune response, the nearest neighbor analysis for phenotype pairs was carried out to calculate the mean distance from each melanoma cell (stained positive for the melanoma cocktail of antibodies) to the nearest $\mathrm{CD} 3+\mathrm{T}$ lymphocyte. Patients with CD showed a shorter average distance between tumor and $\mathrm{T}$ cells than patients having PD (Fig. 2f). Considering the lymphocyte and melanoma cell dimensions, a $25-30 \mu \mathrm{m}$ distance between cells is indicative of an enhanced probability for cell-to-cell contact [33]. CD patients had a significantly increased percentage of UM cells within a radius of $25 \mu \mathrm{m}$ from CD8+ T cells, as compared to PD patients (Fig. 2g). Although not significant, CD patients also showed a trend for a higher frequency of melanoma cells within a $30 \mu \mathrm{m}$ radius from $\mathrm{CD} 8$ + Granzyme $\mathrm{B}+$ activated CTL than $\mathrm{PD}$ patients (Supplementary Figure 2).

\section{The tumor immune cell contexture differs between liver (LM) and extra-hepatic (EM) UM metastases}

To assess whether differences existed in the microenvironment cell composition of hepatic and extra-hepatic UM metastases, patients were divided into two groups according to the site of liver (LM $n=17$ ) or extrahepatic (EM $n=4$; Table 1) metastasis, to be subsequently analyzed by mIHC. Two patients had both hepatic and extra-hepatic metastases, but they were included in the LM group as only their hepatic metastases were evaluated.

Although the CD3+ $\mathrm{T}$ cell lineage was the most represented in the TIL population in either groups and irrespective of location, LM patients disclosed a higher 

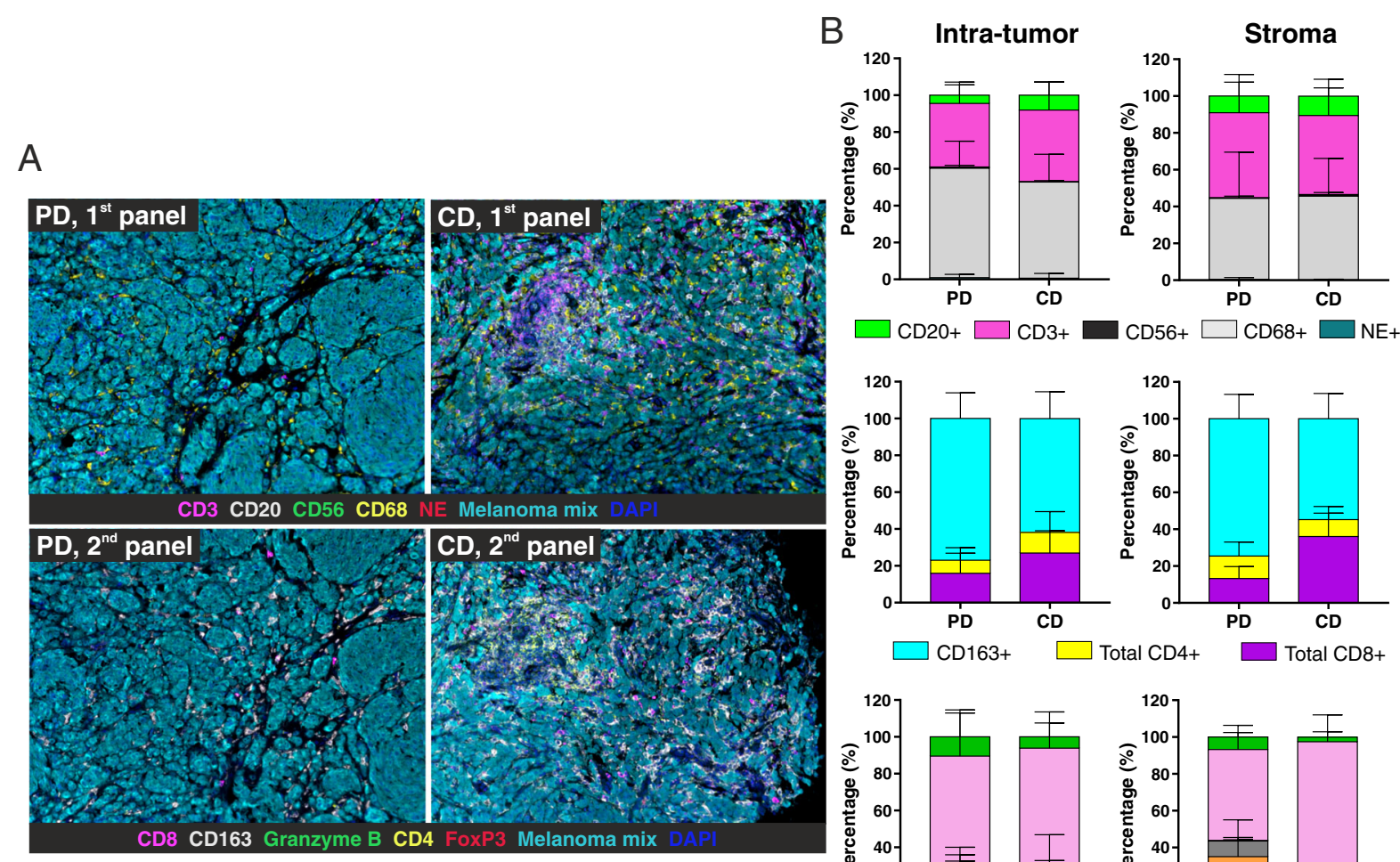

$\square \mathrm{CD} 20+\square \mathrm{CD} 3+\square \mathrm{CD} 56+\square \mathrm{CD} 68+\square \mathrm{NE}+$
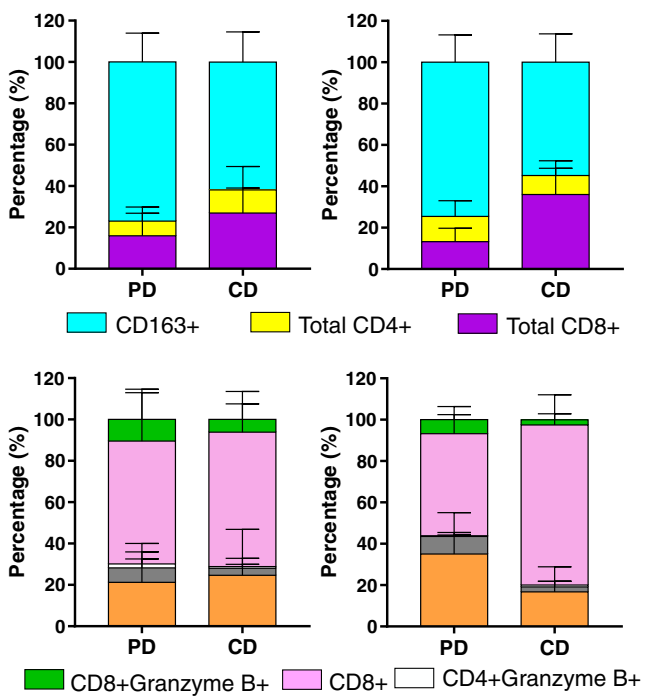
$\square$ CD4+FoxP3+ $\square$ CD4+
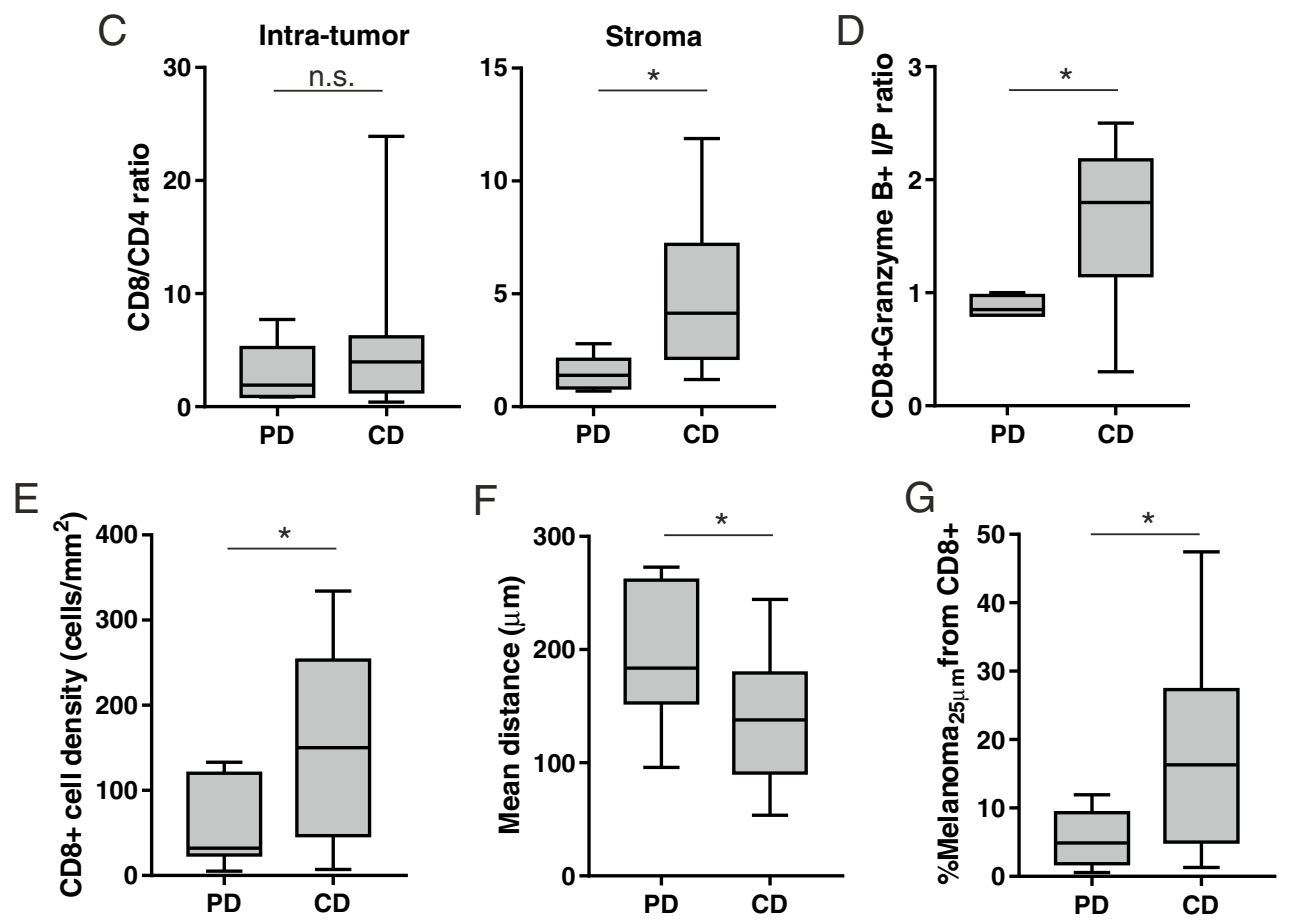

Fig. 2 (See legend on next page.) 
(See figure on previous page.)

Fig. 2 The tumor immune cell composition differs between PD and CD patients. a Representative images of 7-color mIHC staining of metastatic UM samples derived from PD and CD patients. First panel (above): CD3 in magenta, CD20 in white, CD68 in yellow, neutrophil elastase in red, CD56 in green, melanoma mix in cyan, nuclei in blue. Second panel (below): CD4 in yellow, CD8 in magenta, Granzyme B in green, FoxP3 in red, CD163 in white, melanoma mix in cyan, nuclei in blue. Original magnification 20X. b Immune cell quantitation from the two mIHC panels plotted as the percentage of cells in PD and CD patients, calculated in the intra-tumoral and stromal regions. The mean values and the standard deviations are represented. $\mathbf{c}$ CD8+/CD4+ cell ratio calculated in the intra-tumoral and stromal compartments in PD and CD patients. $\mathbf{d}$ Intratumoral/Peri-tumoral (I/P) ratio of CD8 + Granzyme+ T lymphocytes in PD and CD patients. e Intra-tumoral CD8+ T cells density (number of cells/ $\mathrm{mm}^{2}$ ) in PD and CD patients. $\mathbf{f}$ Mean distance $(\mu \mathrm{m}$ ) between each UM cell (stained positive for the melanoma cocktail of antibodies) and the nearest CD3+ T lymphocyte in PD and CD patients. g Percentage of UM cells within a radius of $25 \mu \mathrm{m}$ from CD8+ T lymphocytes in PD and CD patients. Floating box extends from 25th to 75th percentiles, line through the box indicates median, and bars extend from the smallest to largest values. Non-parametric Mann-Whitney statistical analysis was performed across the two groups, and significantly different data are represented by $*(p<0.05)$

percentage of intra-tumor B and T lymphocytes as compared to patients with EM (Fig. 3a, upper panels). These latter, on the other hand, showed a higher percentage of intra-tumoral macrophages (both CD68+ and CD163+; Fig. 3a, upper and middle panels). Whether considering the $\mathrm{T}$ cell composition of the intra-tumoral compartment, in either group of patients total CD8+ cells were more represented than CD4+ cells (Fig. 3a middle panels), but their activation status was different. Indeed, LM patients showed a lower percentage of CD8 + Granzyme $\mathrm{B}+$ lymphocytes and $\mathrm{T}_{\text {reg }}$ cells (Fig. 3a bottom panels). In the peritumoral stroma, patients with LM displayed a 2-fold increased percentage of CD8+ T cells than EM patients, but an about 50\% reduction in CD4+ $\mathrm{T}$ helper cells (Fig. 3a middle panels). As in the intratumoral region, also in the stroma activated CTL and $\mathrm{T}_{\text {reg }}$ cells were less represented in LM as compared to EM (Fig. 3a bottom panels). CD4 + Granzyme B+ T cells and NK cells were negligible (Fig. $3 a$ ).

Quantitative analysis showed that the intra-tumoral densities of CD163+ M2-polarized macrophages and total CD8+ $\mathrm{T}$ cells were higher in patients with $\mathrm{LM}$ as compared to those with EM (Fig. 3b, c). Conversely, the percentage of CD8 + Granzyme B+ activated CTL among the total $\mathrm{CD} 8+$ population within the tumor compartment was lower in LM patients (Fig. 3d).

A direct correlation between $\mathrm{T}$ and $\mathrm{B}$ cells was observed in both the intra-tumoral $(r=0.615,95 \% \mathrm{CI}$ 0.176 to 0.85$)$ and peri-tumoral regions of LM ( $r=$ 0.548 , 95\% CI 0.056 to 0.826; Supplementary Figure 3A). Moreover, also $\mathrm{T}$ cell and macrophage densities were directly proportional in these areas $(r=0.641,95 \% \mathrm{CI}$ 0.217 to 0.861 within the tumor and $r=0.711,95 \% \mathrm{CI}$ 0.319 to 0.895 in the stroma; Supplementary Figure 3B). Of note, when considering only the M2-polarized fraction of macrophages, the association with CTL was observed only within the tumor masses $(r=0.537,95 \% \mathrm{CI}$ 0.06 to 0.814; Supplementary Figure 3C). Furthermore, an inverse correlation between the percentage of activated CTL and the density of CD8+ cells $(r=-0.531$, $95 \% \mathrm{CI}-0.79$ to 0.006$)$ or CD163+ cells $(r=-0.653$,
95\% CI -0.867 to -0.237 ) was disclosed in the intratumoral area (Supplementary Figure 3D). Collectively, these findings suggest that CTL may drive the recruitment of immunosuppressive macrophages to the liver [34], and that this TAM subset may contribute to limit a potentially antitumor immune response in UM-derived LM.

To validate these assumptions, spatial metric analyses were performed and cell-to-cell interactions were assessed. In the nearest neighbor distance analysis, a significant lower mean distance from each melanoma cell and the nearest CD8+ T lymphocyte was observed in patients with LM as compared to patients with metastasis in other sites (Fig. 3e). Based on the quantitative analysis performed on the entire tumor microenvironment area, LM patients displayed a higher percentage of melanoma cells within a $20 \mu \mathrm{m}$ radius from CD8+ CTL (Fig. 3f). However, this group of patients showed also an increased frequency of tumor cells within a $20 \mu \mathrm{m}$ radius from CD163+ M2-polarized macrophages (Fig. 3g), and a higher percentage of $\mathrm{CD} 8+\mathrm{T}$ lymphocytes within a $20 \mu \mathrm{m}$ radius from CD163+ TAM (Fig. 3h). Collectively, these features are highly suggestive of a role for TAM in compromising the antitumor immune response in UM liver metastases.

\section{The immune cells density impacts on overall survival of patients with UM metastases}

Distance analysis carried out in patients based on their status at the last follow-up time point, revealed that alive patients $(n=11)$ had a higher frequency of melanoma cells within a $30 \mu \mathrm{m}$ radius from CD8 + Granzyme B+ CTL, in comparison to dead patients $(n=10$; Table 2 and Supplementary Figure 4). This prompted further exploration on the impact of immune cell subtypes populating the tumor microenvironment on survival.

Based on the median cell densities and cell percentages of each immune cell subset, UM metastases were stratified into high or low tumor-infiltrated groups. The analysis was then performed considering the total area examined (tumor core and peritumoral stroma), the 

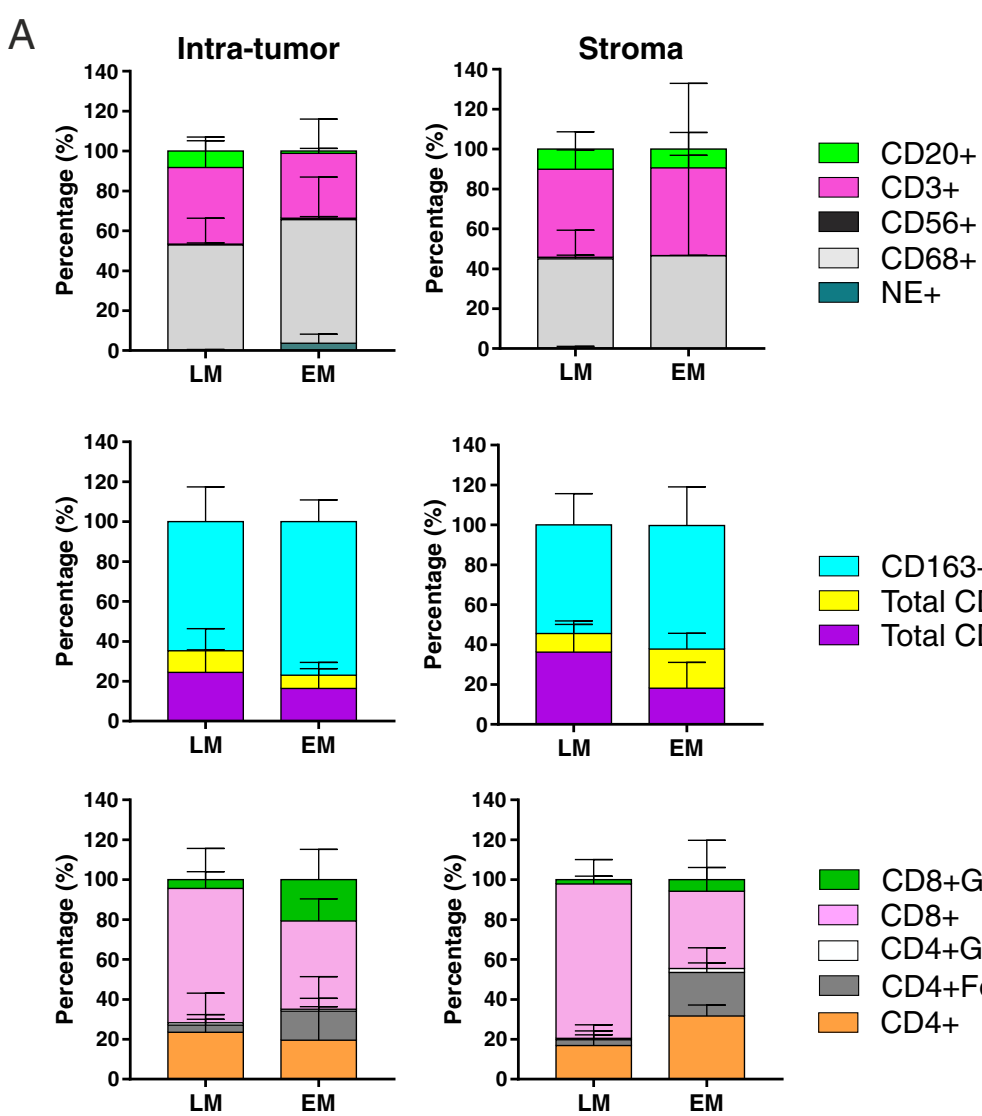

CD163+

Total CD4+

Total CD8+
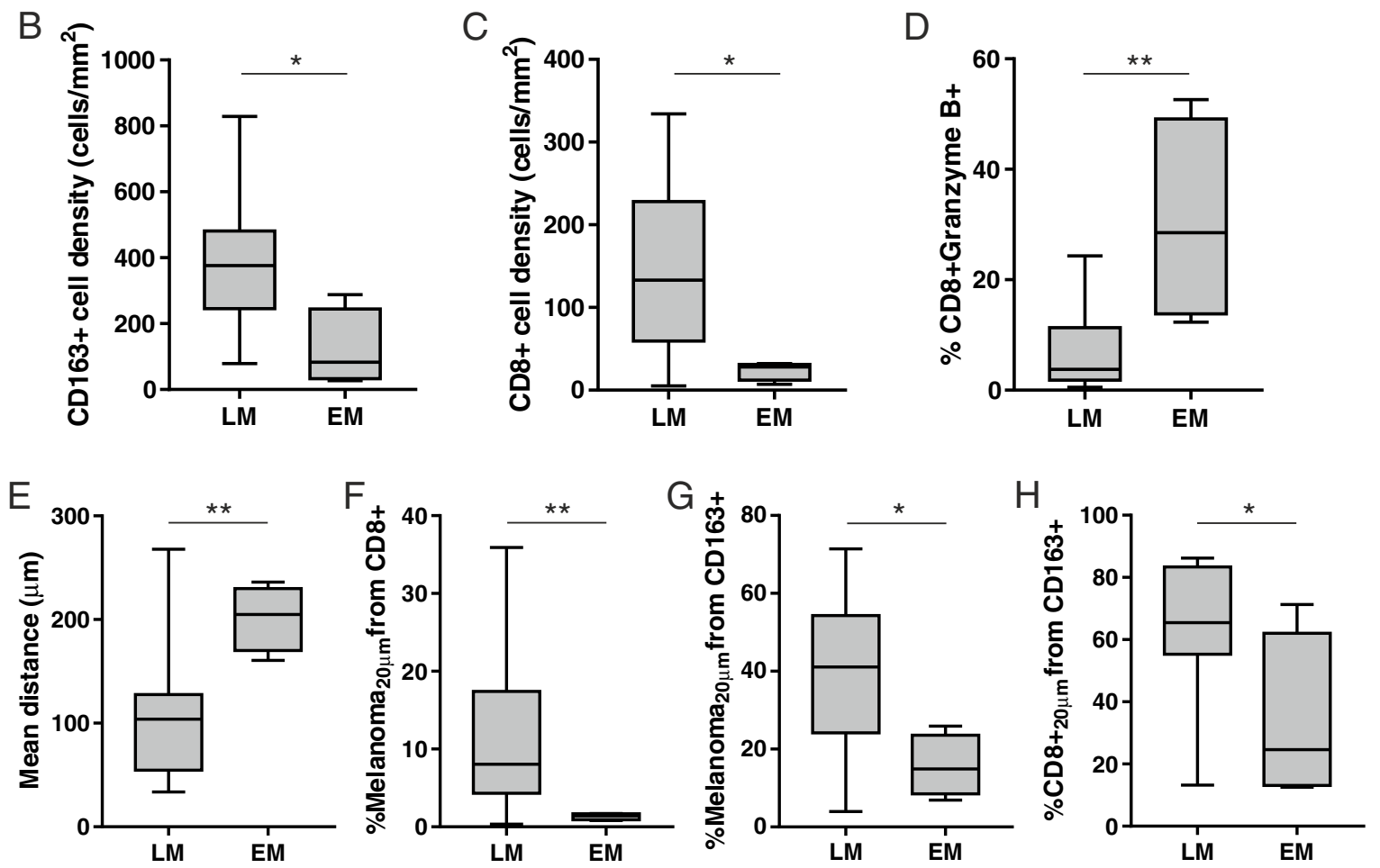

Fig. 3 (See legend on next page.) 


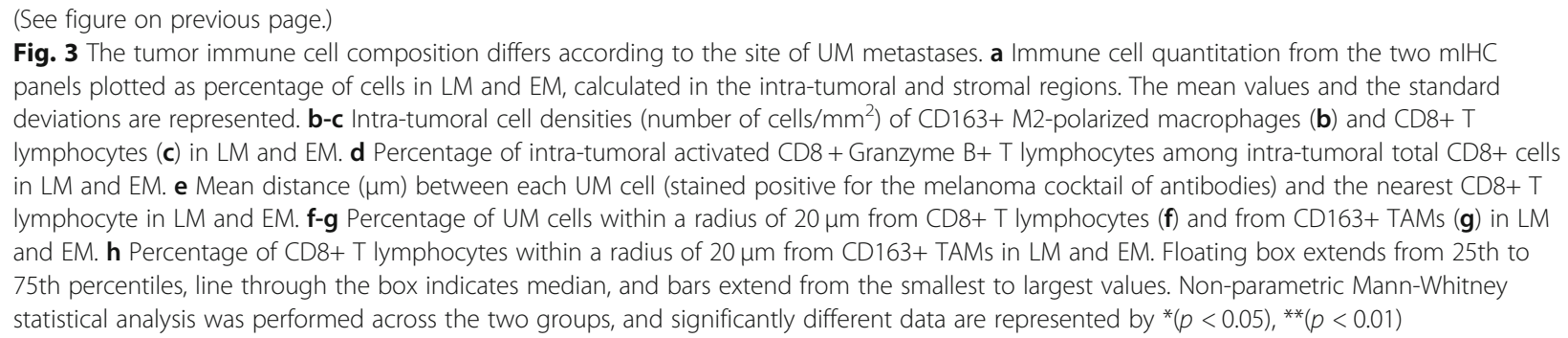

intra-tumoral region only, and the peritumoral stroma only. The density of any infiltrating $\mathrm{T}$ cell or macrophage subtype did not significantly associate with survival (data not shown). Notwithstanding and whether considering the total area of tumor microenvironment, a high percentage of activated CD 8 + Granzyme B+ T lymphocytes among total CD8+ T cells was associated with a significantly prolonged survival (median OS: 135.6 months in the High group vs. 26.2 months in the Low group; HR 0.24, 95\% CI 0.06-0.89, $p=0.022$; Fig. 4a). As we previously reported that also the immune effector cell localization in different areas of tumor microenvironment is an important determinant of patient outcome (Fig. 2), we separately assessed the prognostic value of stromal and intra-tumoral activated CTL. A significant prognostic value was obtained considering only the percentage of intra-tumoral CD8 + Granzyme B+ CTL, as patients with high percentages of such activated CD8+ cells had longer OS (HR $0.23,95 \%$ CI $0.06-0.86, p=$ 0.018; Fig. 4b). These data support the importance of activated CTL tumor infiltration in the prognosis of patients with metastatic UM. Tertiary lymphoid structures (TLS) were also searched and found in 6 out the 21 (28.5\%) metastatic UM samples, but their presence did not associate with OS (Supplementary Figure 5).

Moreover, as the evaluation of the single biomarkers density did not correlate with patient outcome, we explored a combined assessment of both the density and the spatial distribution of the two immune cell populations mainly involved in tumor dynamics, namely $\mathrm{T}$ lymphocytes (CD3+ cells) and macrophages (CD68+ cells). Given the small number of patients analyzed and the observation that the activation of lymphoid and myeloid response correlated each other in patients with CD (Supplementary Figure 1), two groups were created: one consisted of UM metastases with both CD3+ and CD68+ cell densities respectively higher (CD3high/CD68high) or lower (CD3low/CD68low) than the corresponding median values. The second group comprised the UM metastases with discordance between lymphoid and myeloid cell densities (CD3high/CD68low and CD3low/ CD68high). Considering the peritumoral stroma, no significant difference was observed between the two groups (data not shown). Within the tumor region, however, patients with the concordance between $\mathrm{T}$ cell and macrophage densities (CD3high/CD68high and CD3low/ CD68low) exhibited a significant prolonged OS compared to patients with CD3high/CD68low and CD3low/ CD68high (median OS: 96 months vs 10.2 months, respectively; HR 0.12, 95\% CI 0.003-4.73, $p=0.0008$; Fig. 4c). These results highlight the requirement for a balanced lymphoid and myeloid response in the tumor microenvironment of metastatic UM for a better outcome.

Moreover, in an attempt to provide a more comprehensive picture about the importance of the amount of the $\mathrm{T}$ cell infiltrate in relation to survival, a temptative Immunoscore was generated by considering the median densities of CD3+ and CD8+ T cell subtypes in both the intra-tumoral region and the peri-tumoral stroma. $\mathrm{Pa}$ tients with infiltrating $\mathrm{T}$ lymphocyte densities lower than the corresponding median value were scored as Immunoscore 0 (I0), while those with both $\mathrm{CD} 3+$ and $\mathrm{CD} 8+$ cell densities within tumor and stroma regions higher than the median values were scored as Immunoscore 4 (I4). The remaining patients with an Immunoscore ranging from 1 to 3 were grouped and scored as Intermediate Immunoscore (IntI). Kaplan-Meier curves showed three distinct patient groups with statistical differences in OS ( $p=0.011$; Fig. $4 d$ ). Notably, patients with IntI experienced the worst outcome, with a median survival of 12 months when compared to patients with I0 (median survival: 135.6 months, HR 5.0, 95\% CI 1.32-18.8, $p=$ 0.015 ) and those with I4 (HR 5.9, 95\% CI 1.48-23.9, $p=$ 0.044; Fig. 4d).

\section{The tumor immune contexture is associated with overall survival in immunotherapy-treated patients}

In the study cohort, 13 patients (61.9\%) were treated with checkpoint inhibitors immunotherapy, while 8 patients $(38.1 \%)$ with other targeted/systematic therapies (Table 2). No difference in the OS was observed between the two groups $(p=0.43)$, although immunotherapytreated patients displayed a significantly lower density of $\mathrm{CD} 4+$ FoxP3+ $\mathrm{T}_{\text {reg }}$ lymphocytes both in the intratumoral and stromal regions, as compared to individuals receiving other treatments (Fig. 5a). Moreover, within the tumor region, they had a lower density of total 

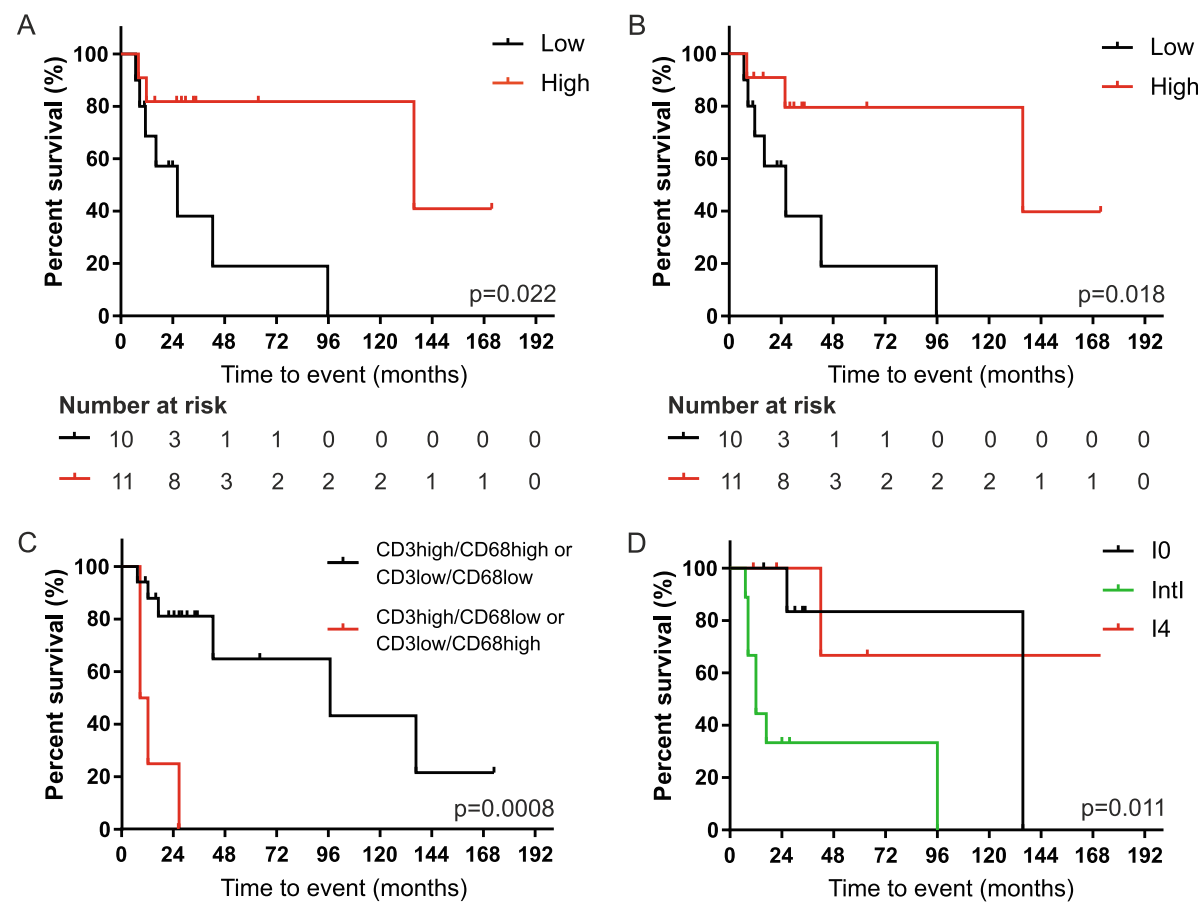

Number at risk

Number at risk

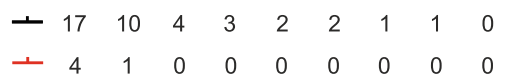

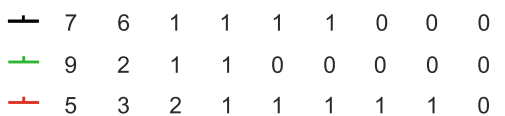

Fig. 4 Effect of immune cell infiltrates on overall survival of metastatic UM patients. a-b Kaplan-Meier curves for overall survival according to the percentage of activated CD8 + Granzyme B+ T lymphocytes among the total CD8+ cells, calculated in the total area (a) or only in the intratumoral region (b). The median cutoff of CD8 + Granzyme B+ percentage was used to separate high and low infiltrate groups. c Kaplan-Meier curves for overall survival according to the combination of intra-tumoral CD3+ T lymphocytes and CD68+ macrophages densities. The median cutoff of each immune cell subset density was used to separate high and low infiltrate. $\mathbf{d}$ Evaluation of the Immunoscore as a prognostic biomarker in metastatic UM. Kaplan-Meier curves for overall survival according to Immunoscore: Immunoscore 0 (I0, $n=7)$; Immunoscore 1,2,3 (Intl, $n=9)$; Immunoscore $4(14, n=5)$. Log-rank statistics were performed to determine significance, $p$ values and the number of patients at risk for each time point are reported in each graph

CD4+ cells (Fig. 5b) and a lower CD4 + FoxP3+/CD8 + Granzyme $\mathrm{B}+$ cell ratio (Fig. $5 \mathrm{c}$ ). Whether considering only the proportion of patients treated with immunotherapies, we additionally observed that those still alive at the end of the follow-up had a lower percentage of $\mathrm{T}_{\text {reg }}$ cells both in the total area and in the intra-tumoral region only (Fig. 5d), and a lower CD4 + FoxP3+/CD8 + Granzyme $\mathrm{B}+$ cell ratio (Fig. 5e). Finally, in immunotherapy-treated patients the low density of stromal $\mathrm{T}_{\text {reg }}$ cells (Fig. 5f), the low stromal $\mathrm{T}_{\text {reg }} / \mathrm{CD} 8+$ cell ratio (Fig. 5g), and the low percentage of $\mathrm{T}_{\text {reg }}$ cells among total CD4+ T lymphocytes both at stromal and intra-tumoral level (Fig. 5h), are all features associated with a prolonged OS. Collectively, these observations suggest that CD4 + FoxP3 $+\mathrm{T}_{\text {reg }}$ cells appear a crucial population for response to immunotherapy.

\section{Discussion}

Currently, the present study comprises one of the largest sample cohort in which the metastatic UM immune microenvironment has been quantitatively analyzed, and in which the frequency and composition of immune cell infiltrate is correlated with patient outcome. Moreover, also the cell topography and thereby the probability of cell-to-cell interactions has been investigated, with additional correlations to clinical and prognostic parameters.

The eye is considered an immune-privileged site, and the immunobiology of primary UM has been already object of extensive investigation [21, 23, 35, 36]. However, only few studies have focused on the characterization of the immune infiltrate and microenvironment of metastatic UM [24, 25, 37], mainly due to the limited availability of biological samples, and because the vast majority of patients are not qualified for surgical resection due to the number or distribution of lesions [38]. This aspect is critical for single-marker IHC studies, which often lead to incomplete quantitative data due to the exhaustion of the FFPE blocks [39]. Multispectral imaging performed on metastatic UM samples allowed us to objectively assess seven markers simultaneously, to 


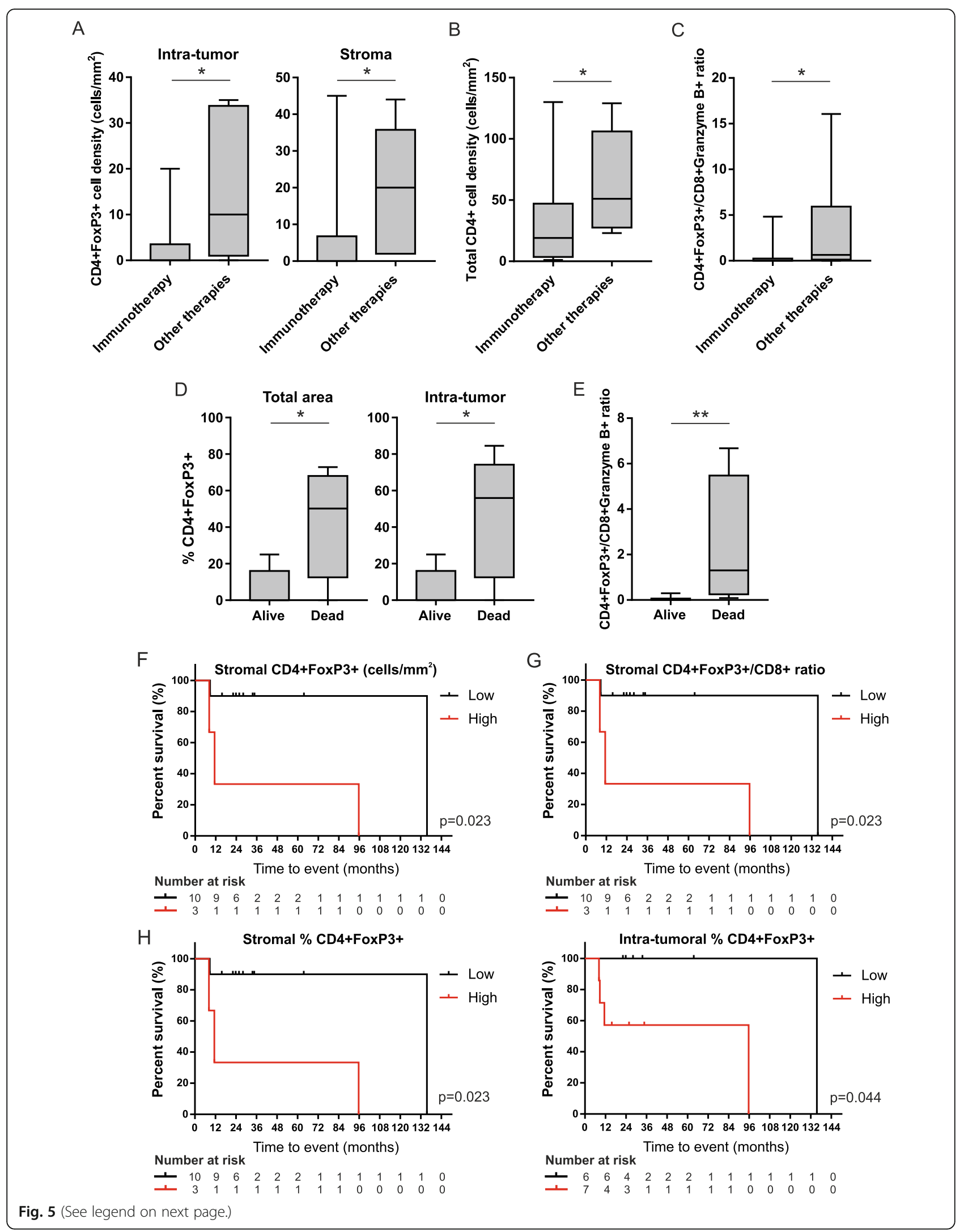


(See figure on previous page.)

Fig. 5 The impact of the tumor immune contexture on overall survival of immunotherapy-treated patients. a-c Comparison between immunotherapy-treated patients and individuals receiving other therapies in terms of (a) intra-tumoral and stromal CD4 + FoxP3 $+T_{\text {reg }}$ cell densities (number of cells $/ \mathrm{mm}^{2}$ ), (b) intra-tumoral total CD4+ T cell density (number of cells $/ \mathrm{mm}^{2}$ ), and (c) intra-tumoral CD4 + FoxP3+/CD8 + Granzyme B+ cell ratio. $\mathbf{d}$ Percentage of CD4 + FoxP3+ cells among total CD4+ lymphocytes, and (e) CD4 + FoxP3+/CD8 + Granzyme B+ cell ratio in the total area of immunotherapy-treated patients who were alive or death at the end of the follow-up. Floating box extends from 25th to 75 th percentiles, line through the box indicates median, and bars extend from the smallest to largest values. Non-parametric Mann-Whitney statistical analysis was performed across the two groups, and significantly different data are represented by $*(p<0.05)$ and $* *(p<0.01)$. f-h Kaplan-Meier overall survival curves in immunotherapy-treated patients according to (f) the stromal CD4 + FoxP3+ cell density, $\mathbf{g}$ the stromal CD4 + FoxP3+/ CD8+ cell ratio, and (h) the percentage of CD4 + FoxP3+ cells among total CD4+ lymphocytes calculated in the stromal or intra-tumoral regions. Log-rank statistics were performed to determine significance, $p$ values and the number of patients at risk for each time point are reported in each graph

precisely quantify the number of cells with a specific phenotype and to determine their cartographic coordinates on a single 4- $\mu \mathrm{m}$ FFPE tissue section, thus consuming very few amounts of the precious metastatic UM sample.

Our observations on immune cells infiltrating UM metastases are consistent with recently published studies $[24,25,37]$, but we have additionally found that the immune cell subsets composition differs according to patient response. Indeed, in UM metastases from PD patients we identified immune features suggestive of an impaired antitumor immune response, such as a relevant presence of pro-tumorigenic M2 macrophages and $\mathrm{T}_{\text {reg }}$ cells, a reduced intra-tumoral CTL density and a lower stromal $\mathrm{CD} 8 / \mathrm{CD} 4$ ratio. These results are in line with the observation that NRP1 gene, which is involved in the immune-modulation of $\mathrm{T}_{\text {reg }}$ cells and M2-polarized macrophages, is upregulated in metastatic UM patients with an OS less than 1 year [40]. Moreover, the spatial context of immune cells has been shown to be critical for cancer development [41], since effector cells require close contacts with target cells to exert their cytotoxic antitumor functions. Our observations that the percentage of melanoma cells close to $\mathrm{T}$ cells was higher in $\mathrm{CD}$ than PD patients and that the majority of CD8 + Granzyme $\mathrm{B}+\mathrm{T}$ cells could be detected within the intratumoral region, suggest a specific anti-tumor effector role of CTL that may perform important biological functions in metastatic UM.

The potential importance of this cell subset is also supported by the observation that the percentage of activated CTL acts as a prognostic indicator able to stratify metastatic UM patients with better OS, while the mere density of total CD8+ T lymphocytes did not associate with patient outcome. Of note, when discriminating between the intra-tumoral and peri-tumoral regions, only the percentage of $\mathrm{CD} 8+$ Granzyme $\mathrm{B}+\mathrm{T}$ lymphocytes within the tumor masses retained the prognostic value, supporting the importance of effector cell localization in metastatic UM. All together, these observations go beyond the bias of previous studies that focused only on the overall cell counts using single marker IHC [24, 25], highlighting the clinical relevance and possible functional importance of $\mathrm{T}$ cell infiltration for metastatic UM control.

TLS may support the activation of CTL against tumor cells, as the presence of TLS in melanoma patients was associated with improved outcome [42]. However, in tumors arising in immunologically privileged sites, such us the brain (glioblastoma) and the eye (uveal melanoma), TLS are usually infrequent [43]. In agreement with this observation, we found TLS only in a small proportion of metastatic UM patients, and no prognostic value was observed.

The complexity of mechanisms orchestrating the immune response against metastatic UM is underlined by the observation that a delicate equilibrium exists in patients with better outcome between lymphoid and myeloid cell responses within the tumor region, but not in the peri-tumoral stroma. Our data are in line with what observed by Massi et al. in a cohort of 158 metastatic cutaneous melanoma patients treated with MAPK inhibitors [44]. Paradoxically, high densities of TAMs and TILs in primary UM are associated with a poor prognosis $[21,45]$, likely because of the immunoregulatory influence of the intraocular microenvironment and the macrophage-mediated regulation of angiogenesis and cancer cell migration, which could promote tumor growth. Thus, the association of low densities of both $\mathrm{T}$ lymphocytes and macrophages with a prolonged OS in UM metastases, might be reminiscent of the primary tumor microenvironment. These results suggest that the combined evaluation of the density and spatial distribution of CD3+ and CD68+ cells in metastatic UM patients can be used as a prognostic indicator in metastatic $\mathrm{UM}$.

The Immunoscore has been reported to overcome the classical TNM system in predicting disease-free survival (DFS) and OS in colorectal cancer (CRC) [46]. The definition of an Immunoscore in cutaneous melanoma is still challenging, even though it is currently under evaluation in lymph node metastases from stage III melanoma 
patients [47] and in metastatic tissues from individuals treated with Ipilimumab (the MISIPI study) [48]. In this scenario, we tried to transfer the Immunoscore concept to metastatic UM as a potential prognostic marker. Despite the limited dimension of our cohort, the Immunoscore stratified patients in three distinct groups with statistically significant differences in terms of OS. However, while a low Immunoscore associates with the shortest DFS and OS in CRC patients, were the metastatic UM patients with I0 and I4 to exhibit a significantly increased OS as compared with those having an intermediated Immunoscore. Reasons for this unexpected trend require further investigations in a larger cohort.

Patients with hepatic UM metastases usually experience a worse outcome as compared to individuals with extra-hepatic UM metastases only [10], thus suggesting a role for the microenvironment in UM metastatic progression. In this study, we had the chance to collect not only hepatic but also extra-hepatic UM metastases, and this allowed a comparative analysis of the tumor immune infiltrate between different UM metastatic sites. The liver is considered an immuno-modulatory organ, whose microenvironment could promote UM metastatic growth by protecting melanoma cells from immune surveillance [49]. Differently from what described by Qin et al. [39], we observed differences in the tumor immune cell contexture between hepatic and extra-hepatic UM metastases, supporting the delicate balance between immune elements with anti- or pro-tumorigenic functions in liver UM metastases. Indeed, the high density of intra-tumoral CD8+ T lymphocytes in LM was counterbalanced by an analogous elevated density of CD163+ M2-polarized macrophages, and by a low percentage of CD8 + Granzyme B+ activated CTL. Moreover, although we detected a higher percentage of tumor cells in close proximity to $\mathrm{T}$ lymphocytes as compared to $\mathrm{EM}$, more than $60 \%$ of CTL in LM were in contact with M2polarized macrophages, thus suggesting a role of TAM in suppressing cytotoxic CD8+ T cell functions. Furthermore, we observed that a high percentage of UM cells in liver were adjacent to pro-tumorigenic CD163+ TAMs. In melanoma cells, the expression of particular molecules, such as colony-stimulator factor 1 (CSF1) or $\mathrm{CD} 47$, in response to $\mathrm{T}$-cell derived cytokines represents a conserved and adaptive resistance mechanism involved in disease progression [34]. Indeed, the interaction between CSF1 on melanoma cells and its CSF1 receptor on macrophages shapes the tumor myeloid cell compartment toward immunosuppression by inducing the differentiation and accumulation of M2 TAM. Besides, the binding of CD47 on cancer cells with the inhibitory receptor signal regulatory protein alpha (SIRP $\alpha)$ on TAM suppresses the ability of macrophages to detect and phagocytose tumor cells [50]. Collectively, these features are highly suggestive of a key role of TAM to impair the antitumor immune response in UM liver metastases by inhibiting the activation of CTL that are recruited to the tumor site. These observations could explain the worse outcome of patients with UM-derived LM and could have implications in the treatment modalities of these patients, through the depletion or re-education of TAM [51].

Finally, we also explored the immune populations indicative of a better response to immunotherapy. Despite the low response rate and the negligible impact on survival of checkpoint inhibitors in metastatic UM [52], in our cohort the patients with a better outcome disclosed a lower percentage of $\mathrm{T}_{\text {reg }}$ lymphocytes and a lower $\mathrm{T}_{\text {reg }} / \mathrm{CTL}$ ratio, thus indicating that the amount of $\mathrm{CD} 4+$ FoxP3 $+\mathrm{T}_{\text {reg }}$ cells in metastatic UM might be considered a predictive biomarker for the response to immunotherapy. In support of this hypothesis, the gene expression analysis performed by Qin et al. on pretreatment samples from six immunotherapy-treated metastatic UM patients revealed an upregulation of genes encoding cytokines and molecules of the proinflammatory signal network regulated by IL-13, IL-4 and NF- $\mathrm{kB}$ in non-responding individuals, and the upregulation of IFN- $\gamma$-regulated genes (SOCS1 and MHC) in responding patients [39].

We are aware that this study has some limitations. First, although being one of the biggest collections examined to date, the cohort is still limited. This aspect, together with the most recent case history, could have influenced the longer median OS observed in our cohort, as compared to what found in literature $[8,9]$. Second, patients were differently treated after metastatic UM diagnosis due to the different available treatments during the accrual; therefore, a possible influence of treatments on survival could not be ruled out. However, the absence of standard effective therapies should exclude or limit the treatment effect. Third, the expression of immune checkpoint molecules was not assessed, mainly due to the very limited availability of material samples. In this regard, however, there is increasing evidence that UM metastases are characterized by reduced levels of PD-1+ lymphocytes and PD-L1-expressing cells as compared to cutaneous melanoma metastases [37, 39, 53], and this can provide a potential explanation for the failure of immunotherapy in UM $[27,52]$. On the other hand, molecular profiling at single cell-resolution on a limited number of UM liver metastases, showed the expression of TIM3, LAG-3, and to some extent, TIGIT receptors on TILs, thus suggesting that alternative immune checkpoints may play a role in $\mathrm{T}$ cell response inhibition $[36,54]$.

\section{Conclusions}

In conclusion, we demonstrated that a mIHC approach provides a meaningful opportunity to study the 
interactions and spatial relationships between tumor and different immune cell types, and to describe the complex landscape of metastatic microenvironment in UM, thus helping to identify more effective and personalized treatment strategies. In this study, we correlated the immune composition of UM metastases microenvironment with the disease control rate, the site of metastasis and patient outcome (Supplementary Figure 6). In particular, we observed that i) the immune cell subsets composition differs according to patient response, highlighting the clinical relevance and possible functional importance of $\mathrm{T}$ cell infiltration for metastatic UM control (Supplementary Figure 6B). ii) A delicate balance exists between immune elements with anti- or pro-tumorigenic functions in liver UM metastases, which could promote UM metastatic growth by protecting melanoma cells from immune surveillance, ultimately explaining the worse outcome of patients with liver metastasis from UM (Supplementary Figure 6C). iii) The percentage and the tumor localization of activated cytotoxic $\mathrm{T}$ lymphocytes act as a prognostic indicator able to stratify metastatic UM patients with better OS (Supplementary Figure 6D). iv) Finally, CD4 + FoxP3+ T cells appear a crucial population for response to immunotherapy (Supplementary Figure 6E).

\footnotetext{
Abbreviations

UM: Uveal melanoma; OS: Overall survival; CTLA-4: Cytotoxic Tlymphocyte antigen 4; PD-1: Programmed cell death protein 1; TILs: Tumor-infiltrating lymphocytes; TAMs: Tumor-associated macrophages; FFPE: Formalin-fixed paraffin-embedded; mIHC: Multiplex immunohistochemistry; DCR: Disease control rate; HR: Hazard ratio; $\mathrm{Cl}$ : Confidence interval; LDH: Lactate dehydrogenase; DEBIRI-

TACE: Transcatheter arterial chemoembolization with microbeads charged with irinotecan; PD: Progressive disease; SD: Stable disease; PR: Partial response; CR: Complete response; CD: Controlled disease; NK: Natural killer; $\mathrm{T}_{\text {reg: }} \mathrm{T}$ regulatory; CTL: Cytotoxic $\mathrm{T}$ lymphocytes; LM: Liver metastasis; EM: Extra-hepatic metastasis; 10: Immunoscore 0; 14: Immunoscore 4; Intl: Intermediate Immunoscore; MAPK: Mitogenactivated protein kinase; TNM: Tumor, node, metastasis; CRC: Colorectal cancer; DSF: Disease-free survival; CSF1: Colony-stimulator factor 1; SIRPa: Signal regulatory protein alpha; NRP1: Neuropilin 1;

SOCS1: Suppressor of cytokine signaling 1; MHC: Major histocompatibility complex
}

\section{Supplementary Information}

The online version contains supplementary material available at https://doi. org/10.1186/s13046-021-01947-1

Additional file 1: Supplementary Figure 1. Correlation analyses. Correlation between different immune cell infiltrates in PD (A) and CD (B-E) patients, within the tumor and in the stroma regions. Data are represented in a scatter plot with the best fit shown as solid line. The non-parametric Spearman's correlation coefficient $(r)$ and $p$ value were calculated for each graph. Supplementary Figure 2. Percentage of UM cells within a radius of $30 \mu \mathrm{m}$ from CD8 + Granzyme B+T lymphocytes in $P D$ and $C D$ patients. Floating box extends from 25th to 75th percentiles, line through the box indicates median, and bars extend from the smallest to largest values. Non-parametric Mann-Whitney statistical analysis was performed across the two groups. Supplementary Figure 3. Correlation analyses. Correlation between different immune cell infiltrates in LM within the tumor and in the stroma regions. Data are represented in a scatter plot with the best fit shown as solid line. The non-parametric Spearman's correlation coefficient ( $r$ ) and $p$ value were calculated for each graph. Supplementary Figure 4. Percentage of UM cells within a radius of $30 \mu \mathrm{m}$ from CD8 + Granzyme B+ T lymphocytes in patients alive and dead at the last follow-up time point. Floating box extends from 25th to 75th percentiles, line through the box indicates median, and bars extend from the smallest to largest values. Non-parametric Mann-Whitney statistical analysis was performed across the two groups, and significantly different data is represented by ${ }^{*}(p<0.05)$. Supplementary Figure 5 . The presence of TLS did not correlate with metastatic UM patient's prognosis. A) Representative 7-color $\mathrm{mIHC}$ image of a TLS found in a metastatic UM sample. Markers and color code are indicated under the picture. Original magnification 20X. B) Kaplan-Meier curves for overall survival according to the presence or absence of TLS in the tumor microenvironment. Logrank statistics were performed to determine significance, $p$ value and the number of patients at risk for each time point are reported. Supplementary Figure 6. A schematic cartoon illustrating the key findings of the manuscript.

\section{Acknowledgements}

Not applicable.

\section{Authors' contributions}

AT, AR and VCS conceived the study. CA, JP, AF and VCS collected biopsies, treated and followed the patients. RC, MS, SB and APDT reviewed the cases and collected tumor samples. AT and VR performed multiplex staining. AT, AR and VCS analyzed and interpreted the data. PDB revised the statistical analyses. AT, AR and VCS drafted the manuscript. All authors contribute to and approved the final version of the manuscript.

\section{Funding}

The research was funded by Fondazione AIRC under IG $2018-I D$. 21354 project - P.I. Rosato Antonio; 5 per Mille 2019 - ID. 22759 program - G.L. Rosato Antonio; BIOV19ROSATO from 5 per Mille 2019, Veneto Institute of Oncology IOV-IRCCS, the Ministry of Health-Alliance Against Cancer (MoH-ACC) project "Research project on CAR T cells for hematological malignancies and solid tumors" RCR-2019-23669115, and the MoH-ACC project ACC WG6 Immunoterapia WG6-ACC-2020, to AR.

\section{Availability of data and materials \\ Not applicable.}

\section{Declarations}

Ethics approval and consent to participate

The study was approved by local Ethics Committee.

\section{Consent for publication}

All authors agreed with submission of the manuscript for publication and agree to be accountable for all aspect of the manuscript.

\section{Competing interests}

The authors declare that they have no competing interests.

\section{Author details}

${ }^{1}$ Department of Surgery, Oncology and Gastroenterology, University of Padova, Padova, Italy. ${ }^{2}$ Pathological Anatomy Unit, Padova University Hospital, Padova, Italy. ${ }^{3}$ Department of Medicine (DIMED), Surgical Pathology Unit, University of Padova, Padova, Italy. ${ }^{4}$ Immunology and Molecular Oncology Diagnostics, Veneto Institute of Oncology IOV-IRCCS, Via Gattamelata 64, 35128 Padova, Italy. ${ }^{5}$ Departement of Diagnostic \& Interventive Radiology-Pederzoli Hospital, Peschiera, VR, Italy. ${ }^{6}$ Melanoma Oncology Unit, Veneto Institute of Oncology IOV-IRCCS, Padova, Italy. ${ }^{7}$ Department of Oncology, Veneto Institute of Oncology IOV-IRCCS, Padova, Italy. ${ }^{8}$ Clinical Research Unit, Veneto Institute of Oncology IOV-IRCCS, Padova, Italy. 


\section{Received: 2 February 2021 Accepted: 13 April 2021}

\section{Published online: 04 May 2021}

\section{References}

1. Carvajal RD, Schwartz GK, Tezel T, Marr B, Francis JH, Nathan PD. Metastatic disease from uveal melanoma: treatment options and future prospects. $\mathrm{Br} J$ Ophthalmol. 2017;101(1):38-44. https://doi.org/10.1136/bjophthalmol-2016-3 09034.

2. Johansson $P$, et al. Deep sequencing of uveal melanoma identifies a recurrent mutation in PLCB4. Oncotarget. 2016;7(4):4624-31. https://doi. org/10.18632/oncotarget.6614.

3. Akbani R, Akdemir KC, Aksoy BA, Albert M, Ally A, Amin SB, et al. Genomic classification of cutaneous melanoma. Cell. 2015;161(7):1681-96. https://doi. org/10.1016/j.cell.2015.05.044.

4. Griewank KG, van de Nes J, Schilling B, Moll I, Sucker A, Kakavand H, et al. Genetic and clinico-pathologic analysis of metastatic uveal melanoma. Mod Pathol. 2014;27(2):175-83. https://doi.org/10.1038/modpathol.2013.138.

5. Singh AD, Turell ME, Topham AK. Uveal melanoma: trends in incidence, treatment, and survival. Ophthalmology. 2011;118(9):1881-5. https://doi. org/10.1016/j.ophtha.2011.01.040

6. Willson JKV, et al. Assessment of metastatic disease status at death in 435 patients with large choroidal melanoma in the collaborative ocular melanoma study (coms) coms report no. 15. Arch Ophthalmol. 2001;119(5): 670-6. https://doi.org/10.1001/archopht.119.5.670.

7. Lorigan JG, Wallace S, Mavligit GM. The prevalence and location of metastases from ocular melanoma: imaging study in 110 patients. Am J Roentgenol. 1991;157(6):1279-81. https://doi.org/10.2214/ajr.157.6.1950883.

8. Kuk D, Shoushtari AN, Barker CA, Panageas KS, Munhoz RR, Momtaz P, et al. Prognosis of mucosal, Uveal, Acral, Nonacral cutaneous, and unknown primary melanoma from the time of first metastasis. Oncologist. 2016;21(7): 848-54. https://doi.org/10.1634/theoncologist.2015-0522.

9. Krantz BA, Dave N, Komatsubara KM, Marr BP, Carvajal RD. Uveal melanoma: Epidemiology, etiology, and treatment of primary disease. Clin Ophthalmol. 2017;11. Dove Medical Press Ltd:279-89. https://doi.org/10.2147/OPTH. S89591.

10. Gragoudas ES, Egan KM, Seddon JM, Glynn RJ, Walsh SM, Finn SM, et al. Survival of patents with metastases from Uveal melanoma. Ophthalmology. 1991;98(3):383-90. https://doi.org/10.1016/S0161-6420(91)32285-1.

11. Hsueh EC, Essner R, Foshag LJ, Ye X, Wang H-J, Morton DL. Prolonged survival after complete resection of metastases from intraocular melanoma. Cancer. 2004;100(1):122-9. https://doi.org/10.1002/cncr.11872.

12. Agarwala SS, Eggermont AMM, O'Day S, Zager JS. Metastatic melanoma to the liver: a contemporary and comprehensive review of surgical, systemic, and regional therapeutic options. Cancer. 2014;120(6):781-9. https://doi. org/10.1002/cncr.28480.

13. Chattopadhyay $C$, et al. Uveal melanoma: From diagnosis to treatment and the science in between. Cancer. 2016;122(15) John Wiley and Sons Inc: 2299-312. https://doi.org/10.1002/cncr.29727.

14. Kinsey EN, Salama AKS. Metastatic uveal melanoma-A review of current therapies and future directions. Eur Oncol Haematol. 2017;13(2) Touch Briefings:100-6. https://doi.org/10.17925/OHR.2017.13.02.100.

15. Danielli R, Ridolfi R, Chiarion-Sileni V, Queirolo P, Testori A, Plummer R, et al. Ipilimumab in pretreated patients with metastatic uveal melanoma: safety and clinical efficacy. Cancer Immunol Immunother. 2012;61(1):41-8. https:// doi.org/10.1007/s00262-011-1089-0.

16. Heppt MV, et al. Combined immune checkpoint blockade for metastatic uveal melanoma: a retrospective, multi-center study. J Immunother Cancer. 2019;7(1):299. https://doi.org/10.1186/s40425-019-0800-0.

17. Chandran SS, Somerville RPT, Yang JC, Sherry RM, Klebanoff CA, Goff SL, et al. Treatment of metastatic uveal melanoma with adoptive transfer of tumour-infiltrating lymphocytes: a single-Centre, two-stage, single-arm, phase 2 study. Lancet Oncol. 2017;18(6):792-802. https://doi.org/10.1016/ S1470-2045(17)30251-6.

18. Onken MD, Worley LA, Char DH, Augsburger JJ, Correa ZM, Nudleman E, et al. Collaborative ocular oncology group report number 1: prospective validation of a multi-gene prognostic assay in uveal melanoma. Ophthalmology. 2012;119(8):1596-603. https://doi.org/10.1016/j.ophtha.2012. 02.017.

19. Field MG, Decatur $C L$, Kurtenbach $\mathrm{S}$, Gezgin $\mathrm{G}$, van der Velden PA, Jager MJ, et al. PRAME as an independent biomarker for metastasis in uveal melanoma. Clin Cancer Res. 2016;22(5):1234-42. https://doi.org/10.1158/1 078-0432.CCR-15-2071.

20. Gooden MJM, De Bock GH, Leffers N, Daemen T, Nijman HW. The prognostic influence of tumour-infiltrating lymphocytes in cancer: a systematic review with meta-analysis. Br J Cancer. 2011;105(1) Nature Publishing Group:93-103. https://doi.org/10.1038/bjc.2011.189.

21. De Cruz POL, Specht CS, McLean IW. Lymphocytic infiltration in uveal malignant melanoma. Cancer. 1990;65(1):112-5. https://doi.org/10.1002/1 097-0142(19900101)65:1<112::AID-CNCR2820650123>3.0.CO;2-X.

22. "Immunohistochemistry of infiltrating lymphocytes in uveal malignant melanoma. | IOVS | ARVO Journals." https://iovs.arvojournals.org/article.a spx?articleid=2161143 (Accessed 01 Oct 2020).

23. Bronkhorst IHG, Ly LV, Jordanova ES, Vrolijk J, Versluis M, Luyten GPM, et al. Detection of M2-macrophages in uveal melanoma and relation with survival. Investig Ophthalmol Vis Sci. 2011;52(2):643-50. https://doi.org/10.11 67/iovs.10-5979.

24. Krishna Y, McCarthy C, Kalirai H, Coupland SE. Inflammatory cell infiltrates in advanced metastatic uveal melanoma. Hum Pathol. 2017;66:159-66. https:// doi.org/10.1016/j.humpath.2017.06.005.

25. Rothermel LD, Sabesan AC, Stephens DJ, Chandran SS, Paria BC, Srivastava AK, et al. Identification of an immunogenic subset of metastatic Uveal melanoma. Clin Cancer Res. 2016;22(9):2237-49. https://doi.org/10.1158/1 078-0432.CCR-15-2294.

26. Augsburger JJ, Corrêa ZM, Shaikh AH. Effectiveness of treatments for metastatic Uveal melanoma. Am J Ophthalmol. 2009;148(1):119-27. https:// doi.org/10.1016/j.ajo.2009.01.023.

27. Khoja L, Atenafu EG, Suciu S, Leyvraz S, Sato T, Marshall E, et al. Metaanalysis in metastatic uveal melanoma to determine progression free and overall survival benchmarks: an international rare cancers initiative (IRCI) ocular melanoma study. Ann Oncol. Aug. 2019;30(8):1370-80. https://doi. org/10.1093/annonc/mdz176.

28. Terai M, Mastrangleo MJ, Sato T. Immunological aspect of the liver and metastatic uveal melanoma. J Cancer Metastasis Treat. 2017;3(10):231. https://doi.org/10.20517/2394-4722.2017.39.

29. Narducci MG, Tosi A, Frezzolini A, Scala E, Passarelli F, Bonmassar L, et al. Reduction of T lymphoma cells and immunological invigoration in a patient concurrently affected by melanoma and Sezary syndrome treated with Nivolumab. Front Immunol. 2020;11:2452. https://doi.org/10.3389/fimmu.202 0.579894

30. Eisenhauer EA, Therasse P, Bogaerts J, Schwartz LH, Sargent D, Ford R, et al. New response evaluation criteria in solid tumours: revised RECIST guideline (version 1.1). Eur J Cancer. 2009;45(2):228-47. https://doi.org/10.1016/j.ejca.2 008.10.026.

31. Wang K, Shen T, Siegal GP, Wei S. The CD4/CD8 ratio of tumor-infiltrating lymphocytes at the tumor-host interface has prognostic value in triplenegative breast cancer. Hum Pathol. 2017;69:110-7. https://doi.org/10.1016/j. humpath.2017.09.012

32. Shah $W$, Yan $X$, Jing $L$, Zhou $Y$, Chen $H$, Wang $Y$. A reversed CD4/CD8 ratio of tumor-infiltrating lymphocytes and a high percentage of CD4+FOXP3+ regulatory $T$ cells are significantly associated with clinical outcome in squamous cell carcinoma of the cervix. Cell Mol Immunol. 2011;8(1):59-66. https://doi.org/10.1038/cmi.2010.56.

33. Carstens JL, et al. Spatial computation of intratumoral T cells correlates with survival of patients with pancreatic cancer. Nat Commun. 2017;8. https://doi. org/10.1038/ncomms15095.

34. Neubert NJ, et al. T cell-induced CSF1 promotes melanoma resistance to PD1 blockade. Sci Transl Med. 2018;10(436). https://doi.org/10.1126/scitra nslmed.aan3311.

35. Basile MS, et al. Immunobiology of uveal melanoma: state of the art and therapeutic targets. Front Oncol. 2019;9. Frontiers Media S.A. https://doi. org/10.3389/fonc.2019.01145.

36. Triozzi PL, Schoenfield L, Plesec T, Saunthararajah Y, Tubbs RR, Singh AD. Molecular profiling of primary uveal melanomas with tumor-infiltrating lymphocytes. Oncoimmunology. 2019;8(10):e947169. https://doi.org/10.41 61/21624011.2014.947169.

37. Qin Y, et al. Parallel profiling of immune infiltrate subsets in uveal melanoma versus cutaneous melanoma unveils similarities and differences: a pilot study. Oncoimmunology. 2017;6(6). https://doi.org/10.1080/2162402 X.2017.1321187

38. Aoyama T, Mastrangelo MJ, Berd D, Nathan FE, Shields CL, Shields JA, et al. Protracted survival after resection of metastatic uveal melanoma. Cancer. 
2000;89(7):1561-8. https://doi.org/10.1002/1097-0142(20001001)89:7<1561:.A ID-CNCR21>3.0.CO;2-R

39. Qin Y, et al. Immune profiling of uveal melanoma identifies a potential signature associated with response to immunotherapy. J Immunother Cancer. 2020;8(2). https://doi.org/10.1136/jitc-2020-000960.

40. Bao R, Surriga O, Olson DJ, Allred JB, Strand CA, Zha Y, et al. Transcriptional analysis of metastatic uveal melanoma survival nominates NRP1 as a therapeutic target. Melanoma Res. Feb. 2021;31(1):27-37. https://doi.org/10.1 097/cmr.0000000000000701.

41. Fridman WH, Pagès F, Sauts-Fridman C, Galon J. The immune contexture in human tumours: Impact on clinical outcome. Nat Rev Cancer. 2012;12(4): 298-306. https://doi.org/10.1038/nrc3245.

42. Cabrita R, Lauss M, Sanna A, Donia M, Skaarup Larsen M, Mitra S, et al. Tertiary lymphoid structures improve immunotherapy and survival in melanoma. Nature. 2020;577(7791):561-5. https://doi.org/10.1038/s41586-01 9-1914-8.

43. C. Sautès-Fridman, F. Petitprez, J. Calderaro, and W. H. Fridman, "Tertiary lymphoid structures in the era of cancer immunotherapy," Nat Rev Cancer, vol. 19, no. 6. Nature Publishing Group, pp. 307-325, Jun. 01, 2019, doi: https://doi.org/10.1038/s41568-019-0144-6.

44. Massi $D$, et al. The density and spatial tissue distribution of CD8+ and CD163+ immune cells predict response and outcome in melanoma patients receiving MAPK inhibitors. J Immunother Cancer. 2019;7(1). https://doi.org/1 0.1186/s40425-019-0797-4.

45. Bronkhorst IHG, Khanh Vu TH, Jordanova ES, Luyten GPM, van der Burg SH, Jager MJ. Different subsets of tumor-infiltrating lymphocytes correlate with macrophage influx and monosomy 3 in uveal melanoma. Investig Ophthalmol Vis Sci. 2012;53(9):5370-8. https://doi.org/10.1167/iovs.11-9280.

46. Pagès F, Mlecnik B, Marliot F, Bindea G, Ou FS, Bifulco C, et al. International validation of the consensus Immunoscore for the classification of colon cancer: a prognostic and accuracy study. Lancet. 2018;391(10135):2128-39. https://doi.org/10.1016/S0140-6736(18)30789-X.

47. Capone M, et al. Immunoscore: a new possible approach for melanoma classification. J Immunother Cancer. Dec. 2014;2(S3):P193. https://doi.org/1 0.1186/2051-1426-2-s3-p193.

48. Bifulco C, et al. MISIPI study: Melanoma ImmunoScore evaluation in patients treated with IPllimumab. J Transl Med. 2014;12(Suppl 1):P11. https://doi. org/10.1186/1479-5876-12-s1-p11.

49. Racanelli V, Rehermann B. The liver as an immunological organ. Hepatology. 2006;43(2 SUPPL):1. https://doi.org/10.1002/hep.21060.

50. Yang M, McKay D, Pollard JW, Lewis CE. Diverse functions of macrophages in different tumor microenvironments. Cancer Res. 2018;78(19) American Association for Cancer Research Inc:5492-503. https://doi.org/10.1158/00085472.CAN-18-1367.

51. Kowal J, Kornete M, Joyce JA. Re-education of macrophages as a therapeutic strategy in cancer. Immunotherapy. 2019;11 (8) Future Medicine Ltd:677-89. https://doi.org/10.2217/imt-2018-0156.

52. Algazi AP, Tsai KK, Shoushtari AN, Munhoz RR, Eroglu Z, Piulats JM, et al. Clinical outcomes in metastatic uveal melanoma treated with PD-1 and PDL1 antibodies. Cancer. 2016;122(21):3344-53. https://doi.org/10.1002/cncr.3 0258 .

53. Javed A, Arguello D, Johnston C, Gatalica Z, Terai M, Weight RM, et al. PD-L1 expression in tumor metastasis is different between uveal melanoma and cutaneous melanoma. Immunotherapy. 2017;9(16):1323-30. https://doi.org/1 0.2217/imt-2017-0066.

54. Durante MA, et al. Single-cell analysis reveals new evolutionary complexity in uveal melanoma. Nat Commun. 2020;11(1). https://doi.org/10.1038/s414 67-019-14256-1.

\section{Publisher's Note}

Springer Nature remains neutral with regard to jurisdictional claims in published maps and institutional affiliations.

Ready to submit your research? Choose BMC and benefit from:

- fast, convenient online submission

- thorough peer review by experienced researchers in your field

- rapid publication on acceptance

- support for research data, including large and complex data types

- gold Open Access which fosters wider collaboration and increased citations

- maximum visibility for your research: over $100 \mathrm{M}$ website views per year

At BMC, research is always in progress.

Learn more biomedcentral.com/submissions 